\title{
Overexpression of PIK3CA is associated with lymph node metastasis in esophageal squamous cell carcinoma
}

\author{
ICHIRO AKAGI $^{1}$, MASAO MIYASHITA ${ }^{1}$, HIROSHI MAKINO ${ }^{1}$, TSUTOMU NOMURA ${ }^{1}$, \\ NOBUTOSHI HAGIWARA ${ }^{1}$, KEN TAKAHASHI ${ }^{1}$, KAZUMITSU CHO ${ }^{1}$, TAKUYA MISHIMA ${ }^{2}$, \\ OSAMU ISHIBASHI ${ }^{2}$, TOSHIKAZU USHIJIMA ${ }^{3}$, TOSHIHIRO TAKIZAWA ${ }^{2}$ and TAKASHI TAJIRI ${ }^{1}$ \\ Departments of ${ }^{1}$ Surgery for Organ Function and Biological Regulation and ${ }^{2}$ Molecular Anatomy and Medicine, \\ Nippon Medical School; ${ }^{3}$ Carcinogenesis Division, National Cancer Center Research Institute, Tokyo, Japan
}

Received July 24, 2008; Accepted September 16, 2008

DOI: 10.3892/ijo_00000202

\begin{abstract}
The genomic region containing PIK3CA was found to be amplified in esophageal squamous cell carcinoma (ESCC) tissue. PIK3CA at 3q26, which encodes the $p 110 \alpha$ catalytic subunit of phosphatidylinositol (PI) 3-kinase, is a unique intracellular signal transducer characterized by its lipid substrate specificity. In order to characterize PIK3CA in ESCC, we investigated hot-spot mutations in exons 1, 9 and 20, the copy number gain, the expression levels of mRNA and protein. Analysis in exon 9 of the PIK3CA gene revealed mutation in $7.7 \%$ (4 of 52) of ESCC samples. No mutation was detected in either exon 1 or exon 20. Copy number amplifications of PIK3CA were found in 12 of the 45 patients $(26.7 \%)$. PIK3CA mRNAs were examined in 37 ESCC patients as determined by quantitative RT-PCR and the mean mRNA level of PIK3CA in ESCC tissues was 2.61-fold higher compared with that in corresponding non-tumorous esophageal epithelia $(\mathrm{P}<0.001)$. Immunohistochemically, positive immunoreaction for PIK3CA was detectable in 33 of $66(50.0 \%)$ ESCC cases, while it was not detectable in the remaining 33 cases. Furthermore, comparing the cases with negative staining with those with positive staining for PIK3CA, the presence of node metastasis was significantly correlated with those with positive staining $(\mathrm{P}<0.05)$. This study is the first report providing comprehensive analysis of PIK3CA expression in ESCC. These results indicate that PIK3CA may play a crucial role in the development of ESCC and serve as an indicator for lymph node metastasis.
\end{abstract}

Correspondence to: Dr Ichiro Akagi, Department of Surgery for Organ Function and Biological Regulation, Nippon Medical School 1-1-5 Sendagi, Bunkyo-ku, Tokyo 113-8603, Japan

E-mail: ichiro@nms.ac.jp

Key words: esophageal squamous cell carcinoma, phosphatidylinositol 3-kinase, PIK3CA, immunohistochemistry, RT-PCR, lymph node metastasis

\section{Introduction}

The genetic mechanisms underlying the development and progression of esophageal squamous cell carcinoma (ESCC) are among the most complex in human tumors and it has been reported that gene amplification is one of the essential mechanisms of oncogene activation in many cancers (1). Various chromosomal amplicons have recently been identified in ESCC by comparative genomic hybridization (CGH) (2). In particular, genomic amplification of the $3 q 26$ region was identified to be one of the most frequent amplicons in ESCC (2-6). It would be of great interest to detect the specific gene targets within these amplicons, however, they remain largely unknown.

In search of the genes whose selection drives 3 q26 amplification, a recent study has provided convincing evidence that PIK3CA at 3q26 (7), which encodes the p110 $\alpha$ catalytic subunit of phosphatidylinositol (PI) 3-kinase (Gene Bank NM_006218), is found to be amplified and overexpressed in ovarian and cervical cancer (8-11). The increased copy number of the PIK3CA gene is associated with increased $P I K 3 C A$ transcription, $p 110 \alpha$ protein expression and PI3K activity in ovarian cancer (9). It has been reported that aberrant PI3-kinase activation plays important roles in sustaining processes important to malignancy, including cell proliferation, adhesion, survival and motility $(9,12-18)$. PIK3CA allows Ser/Thr kinase (Akt), which is one critical downstream target of this signaling pathway, to be phosphorylated by PDK1 and PDK2 at Thr308 and Ser473, respectively (12). After phosphorylation by Akt, the oncoprotein Mdm2 binds the transactivation domain of tumor suppressor $p 53$ and inhibits expression of $p 53$-regulated genes involved in cell cycle arrest and apoptosis (12). In addition to Akt, PI3-kinase has been shown to regulate the activity of other cellular targets, such as the serum and glucocorticoid-inducible kinase (SGK), the small GTP-binding proteins RAC1 and CDC42 and protein kinase $\mathrm{C}(\mathrm{PKC})$, in an $A k t$-independent manner through poorly characterized mechanisms (12). The activity of these targets leads to survival, cytoskeletal rearrangement and transformation (12). More recently, somatic mutations of PIK3CA have been reported in carcinomas of the colon, breast, brain, liver, stomach, lung, ovary, head and neck. The 
mutated PIK3CA protein is able to activate Akt in the absence of growth factors (19-21). A recent study has reported on the somatic mutations of PIK3CA in ESCC, however, the incidence is lower than in other malignancies (22). The information on both in vitro data and the clinicopathological relevance of PIK3CA in ESCC is, however, sparse and these findings on PIK3CA in various tumors promoted us to investigate both the genetic alterations of PIK3CA and expression patterns in ESCC which is one of the most clinically malignant neoplasms.

In order to characterize PIK3CA in ESCC, we investigated PIK3CA hot-spot mutations in exons 1, 9 and 20, the copy number gain, the expression of PIK3CA mRNA and protein. Finally, in addition to the International Union Against Cancer Tumor-Node-Metastasis (TNM) classification, clinicopathological factors including age, gender, vascular and lymphatic invasion, intraepithelial spread, intramural metastasis, histological grading, stage and development of recurrence were analyzed.

\section{Materials and methods}

Patients and tissues. Sixty-six representative cases with esophageal squamous cell carcinoma who underwent surgery at Nippon Medical School Main Hospital (Bunkyo-ku, Tokyo, Japan) were selected from our archives. The patients consisted of 53 males and 13 females, whose median age was 65 years (range, 45-81 years). The distribution of tumor grades was stage 0 (4 cases), stage I (19 cases), stage IIA (11 cases), stage IIB (9 cases), stage III (13 cases), stage IVA (5 cases) and stage IVB (5 cases). This study was carried out in accordance with the principles embodied in the Declaration of Helsinki, 1975 and informed consent for the usage of esophageal tissues was obtained from each patient.

DNA extraction and mutation analysis. Genomic DNAs were extracted from both tumor and non-tumorous tissues using a DNeasy tissue kit (Qiagen, Valencia, CA). The corresponding non-tumorous tissues were obtained from surgically resected tissues which were located at least $5 \mathrm{~cm}$ away from the tumors. Genomic DNAs from corresponding non-tumorous tissues were also analyzed to confirm whether the nucleotide substitutions detected in tumor tissues were somatic in nature or not, when nucleotide changes were detected in tumor tissues. Genomic DNAs (100 ng per sample) were amplified with primers covering the entire coding region and the exon/intron boundaries of the desired exons using the web-free software Primer3 (Table IA). Exons 1, 9 and 20 of PIK3CA were sequenced directly using the BigDye Terminator Cycle Sequencing kit (Applied Biosystems, Foster City, CA) and an ABI 3730 automated capillary sequencer. Analyses of all PCR products with sequence variants were repeated for confirmation.

PIK3CA copy number quantitation. The PIK3CA copy number was evaluated by real-time quantitative PCR analysis. Primers were designed for the simultaneous amplification of a $150 \mathrm{bp}$ fragment of PIK3CA gene (3q26) and a 187 bp segment of COL7A1 (3p21), a single copy gene taken as control (Table IB). Gene chosen as control is located on the same chromosome as the target gene to ensure that the increase of value, suggestive of an increase in copy number, is not the result of the corresponding chromosome polysomy. Reactions were performed in $25 \mu \mathrm{l}$ volume containing $80 \mathrm{ng}$ of DNA, $300 \mathrm{nM}$ each primer (for both $P I K 3 C A$ and COL7A1, in independent reactions) and $1 \mathrm{X}$ SYBR-Green PCR Master Mix according to the manufacturer's protocol. PCR universal conditions were: $10 \mathrm{~min}$ at $95^{\circ} \mathrm{C}$, followed by 40 cycles of $95^{\circ} \mathrm{C}$ for $5 \mathrm{sec}$ and $60^{\circ} \mathrm{C}$ for $31 \mathrm{sec}$. Samples were analyzed in triplicate. Each amplification reaction was checked for the absence of nonspecific PCR products by running the dissociation protocol. Reactions were carried out on an ABI PRISM 7300 Sequence Detection System (Applied Biosystems).

Sample DNA quantity (M, mean value from triplicates) was calculated by interpolation. Quantitative-PCR was applied to samples of 45 patients to validate the semiquantitave PCR approach previously described. The relative copy number (Q) of PIK3CA vs. COL7Al gene in a tumor sample (T) with respect to its normal tissue counterpart $(\mathrm{N})$ was calculated using the following equation:

$$
\mathrm{Q}=\frac{q T}{q N}=\frac{M_{\text {PIK } 3 C A}^{T} / M^{T}{ }_{C O L 7 A I}}{M_{\text {PIK3CA }}^{N} / M^{N}{ }_{C O L 7 A I}}
$$

Moreover, the results were divided into 'No amplification' for $<2.0$ relative copy number and 'Amplification' for $\geq 2.0$ (23).

RNA preparation and quantitative RT-PCR. We quantified mRNA levels of PIK3CA using a real-time fluorescence detection method. The number of sample for quantitative RT-PCR corresponded with that for immunohistochemistry except for those samples in which tumor cells were too few to allow extraction of sufficient tumor RNA. Two micrograms of total RNA was reverse-transcribed. Taq Man ${ }^{\circledR}$ primers and probes for human PIK3CA (Taq Man Gene Expression Assay; Applied Biosystems) were obtained and GAPDH (Applied Biosystems) served as a reference, with each sample being normalized on the basis of its GAPDH content.

Cells and culture conditions. Two human esophageal squamous cell carcinoma cell lines: TE-series 1 and 8, were kindly provided by the Cell Resource Center for Biomedical Research, Tohoku University. All cell lines were cultured in RPMI-1640 (Sigma-Aldrich Co., St. Louis, MO), which was supplemented with $100 \mathrm{U} / \mathrm{ml}$ penicillin, $100 \mu \mathrm{g} / \mathrm{ml}$ streptomycin (Gibco) and 10\% fetal bovine serum. Cells were grown as subconfluent mono-layers in a humidified atmosphere containing $5 \% \mathrm{CO}_{2}$ at $37^{\circ} \mathrm{C}$ and passaged using a treatment with $0.25 \%$ trypsin every 7 days.

Immunofluorescence analysis. TE cells grown to $70 \%$ confluence on slide glass were fixed in Zamboni solution at room temperature for $30 \mathrm{~min}$. After a specific antibody against PIK3CA (Santa Cruz Biotechnology, USA) was added for $2 \mathrm{~h}$ at room temperature, Alexa Fluor 594-conjugated anti-goat antibody (Invitrogen Corp., Carlsbad, CA) was used as a second antibody for $60 \mathrm{~min}$ at room temperature. Finally, cells were stained for $10 \mathrm{~min}$ at room temperature with 4,6diamidino-2-phenylindole (DAPI). Pictures were taken using 
Table I. Primer sequences and experimental conditions for PIK3CA analysis.

A, Primer sequences of exons

\begin{tabular}{|c|c|c|c|c|}
\hline Exon & Primer sequence ( $5^{\prime}$ to $\left.3^{\prime}\right)$ & Product length & MW & $\operatorname{Tm}\left({ }^{\circ} \mathrm{C}\right)$ \\
\hline 1 & F: GCCTAATCAAGTCAAACTATGGAA & 24 & 7353.8 & 66 \\
\hline 1 & R: GCTATTTAAGATTACGAAGGTATTGG & 26 & 8064.3 & 70 \\
\hline 9 & F: TGGTTCTTTCCTGTCTCTGAAAA & 23 & 6995.6 & 64 \\
\hline 9 & R: ACATGCTGAGATCAGCCAAA & 20 & 6119.0 & 58 \\
\hline 20 & F: CGAAAGCCTCTCTAATTTTGTG & 22 & 6700.4 & 62 \\
\hline 20 & R: TTTTCATTCTTTTCCAATCAATGT & 24 & 7233.8 & 60 \\
\hline
\end{tabular}

B, Primer sequences of genes

\begin{tabular}{llrr}
\hline Gene & \multicolumn{1}{c}{ Primer sequence (5' to 3') } & Size (bp) & Tm $\left({ }^{\circ} \mathrm{C}\right)$ \\
\hline PIK3CA & F: GAGAGGTTTCAGGAGATGTGT & 150 & 60 \\
& R: GGCTAGGGTCTTTCGAATGTA & 187 & 60 \\
& F: GTAACAGACCTGCAAGCCAC $7 A 1$ & 81 & 60 \\
& R: GAGAGGGCTGGAGGTACAC & 87 & 60 \\
\hline
\end{tabular}

Tm, temperature.

a cooled CCD camera and MetaMorph Imaging Software (Universal Imaging Corporation, Downingtown, PA) with equal exposure times.

Immunohistochemistry for PIK3CA. For PIK3CA staining, immunohistochemical staining was performed using the standard streptavidin-biotin-peroxidase complex (SAB)method (Histofine SAB-PO kit, Nichirei, Tokyo, Japan). The sections were incubated with commercially available goat polyclonal antibody raised against PIK3CA, diluted 1:100 at $4^{\circ} \mathrm{C}$ overnight and then incubated with biotinylated rabbit anti-goat $\mathrm{IgG}+\operatorname{Ig} \mathrm{A}+\mathrm{IgM}$ for $10 \mathrm{~min}$. Finally, they were incubated with streptavidin-biotin-peroxidase complex and were observed with an Olympus DP70 microscope (Olympus Optical, Tokyo, Japan). To exclude background staining by non-specific antibody binding, negative controls were included in each test.

Evaluation of PIK3CA protein expression. The prevalence of immunoreactive PIK3CA in tumors was scored semiquantitatively and blindly by two independent investigators and confirmed by a third investigator. According to a previous study with minor modifications, the staining was scored on a scale from grade 0 to $3+$ as follows: 0 , no staining; $1+,<50 \%$ with weak or strong intensity; $2+, \geq 50 \%$ with weak intensity; and $3+, \geq 50 \%$ with strong intensity (24). For statistical analysis, we classified PIK3CA expression of score 0 or $1+$ as negative and score $2+$ or $3+$ as positive.

Statistical analysis. All of the statistical analyses were performed with Stat-View 4.5 statistical software (SAS Institute, Inc., Cary, NC). All results were expressed as mean $\pm \mathrm{SD}$ and $\mathrm{P}<0.05$ was used for significance. The
Mann-Whitney test was used to assess the mRNA levels between tumor samples and normal samples and either the Mann-Whitney test or Tukey-Kramer test was selected to assess the association of the mRNA levels and clinicopathological parameters. Additionally, either Fisher's exact probability test or the Chi-square test was used to examine a possible association between PIK3CA protein expression and clinicopathological parameters.

\section{Results}

Mutation analysis of PIK3CA. Most of the reported PIK3CA mutations cluster in small regions within the p85 binding (exon 1), helical (exon 9) and kinase (exon 20) domains, with E542K, E545K and H1047R constituting three mutational hot spots. Therefore, the analysis of the PIK3CA gene was restricted to these three exons and we identified $P I K 3 C A$ sequence changes in 4 out of 52 ESCC samples $(7.7 \%)$ (Fig. 1). To confirm whether these sequence changes were somatic mutations or single nucleotide polymorphisms (SNPs) of PIK3CA, we also examined the sequence of corresponding non-tumorous tissues. Because these sequence changes were not detected in the corresponding non-tumorous tissues, these alterations were confirmed as somatic mutations, not SNPs. With respect to exon 9, PIK3CA mutation A1634C was detected in 4 of 52 ESCC samples (Table II). On the other hand, no mutation was detected in either exon 1 or exon 20 .

Copy number analysis of PIK3CA. Copy number amplifications of $P I K 3 C A$ were found in 12 of the 45 patients $(26.7 \%)$ for whose results in both tumorous lesion and normal lesion were available (Fig. 2 and Table III). We found no statistical 
Table II. PIK3CA mutation in esophageal squamous cell carcinoma.

\begin{tabular}{lcccccc}
\hline Case & Gender & Stage & Exon & Domain & Nucleotide & Amino acid \\
\hline 28 & Male & IVA & 9 & Helical & A1634C & E545A \\
36 & Male & IIB & 9 & Helical & A1634C & E545A \\
48 & Male & I & 9 & Helical & A1634C & E545A \\
52 & Male & IVB & 9 & Helical & A1634C & E545A \\
\hline
\end{tabular}

\section{A A TCACT GNGCAGGA}

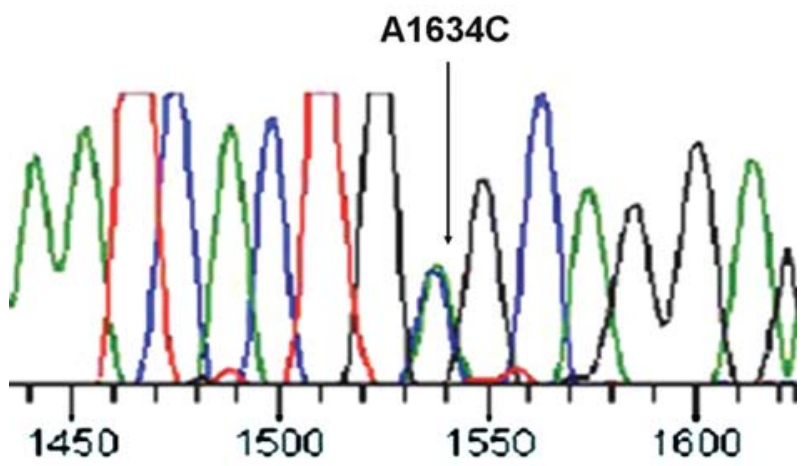

Figure 1. The analysis of the PIK3CA gene was restricted to exon 1, 9 and 20 and we identified PIK3CA sequence changes of exon 9 in 4 out of 52 (7.7\%) ESCCs. The arrow indicates the location of somatic mutation. The nucleotide and amino acid alteration are indicated in the center of this figure. No mutation was detected in either exon 1 or exon 20 .

significance for the correlation between PIK3CA amplification and its protein expression (data not shown).

Evaluation of PIK3CA mRNA status. We used quantitative RT-PCR to assess mRNA levels in ESCC specimens.
Table III. PIK3CA relative copy number by quantitative RT-PCR in ESCC.

\begin{tabular}{lcc}
\hline Gene & $\begin{array}{c}\text { No amplification } \\
(\text { copy number }<2.0)\end{array}$ & $\begin{array}{c}\text { Amplification } \\
(2.0 \leq \text { copy number })\end{array}$ \\
\hline PIK3CA $(\mathrm{n}=45)$ & 33 & 12 \\
\hline
\end{tabular}

Twenty-nine of the 37 ESCC patients (78.4\%) had higher mRNA values in their tumor samples compared with corresponding normal esophageal epithelia. As a whole, the mean mRNA level of PIK3CA in ESCC samples was 2.61fold higher compared with those in corresponding normal esophageal epithelia, as shown in Fig. $3(\mathrm{P}<0.001)$.

Immunohistochemical analysis of PIK3CA. To examine the expression and localization of PIK3CA protein in ESCC, we performed immunohistochemistry. Since the epithelial layer in some normal esophageal tissues showed weak cytoplasmic staining for PIK3CA, it was appropriate to group both no staining and $1+$ staining into the negative group. Positive immunoreaction for PIK3CA was detectable in 33 of 66 $(50.0 \%)$ esophageal squamous cell carcinoma specimens with $>$ grade 2 (Fig. 4A and B), whereas the remaining 33 samples

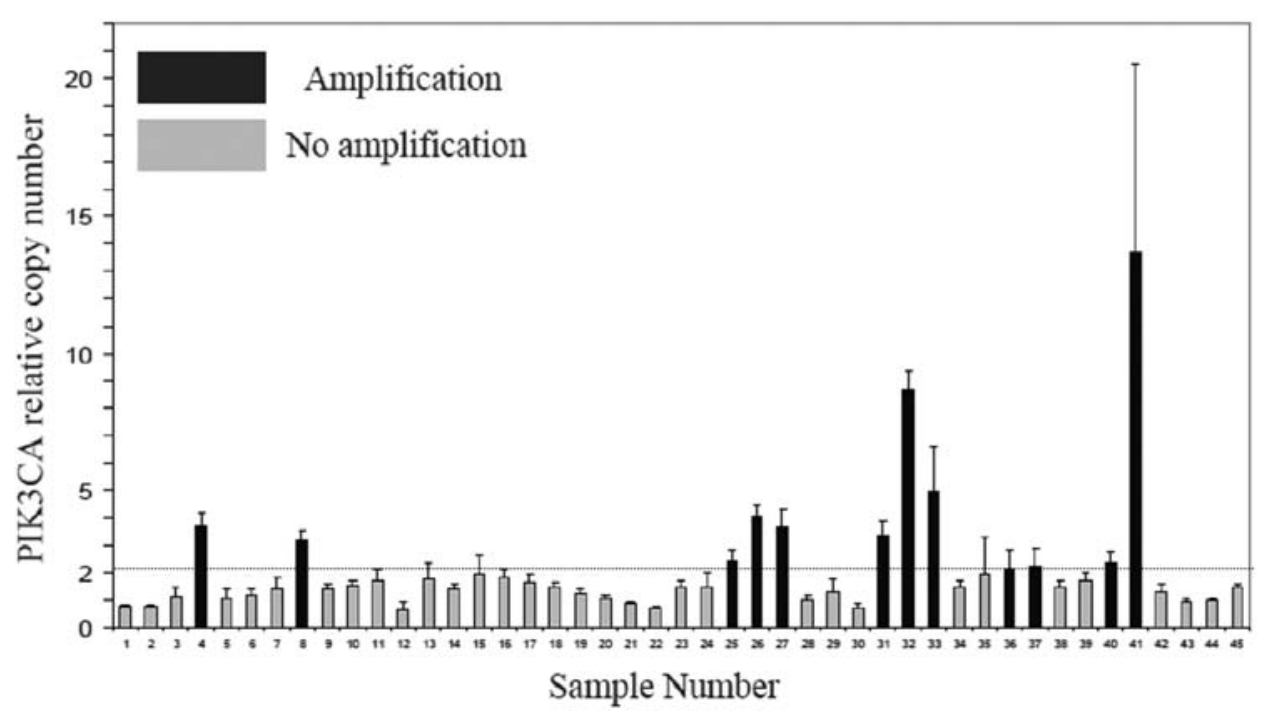

Figure 2. Histogram illustrating PIK3CA relative copy number in 45 samples. The case number is indicated below the panel. The $P I K 3 C A$ copy number ratio $(P I K 3 C A / C O L 7 A 1)$ in tumor samples was divided by that in normal tissue counterparts. The PIK3CA relative copy number $(\geq 2.0)$ was considered amplified $(\mathrm{n}=12)$. 


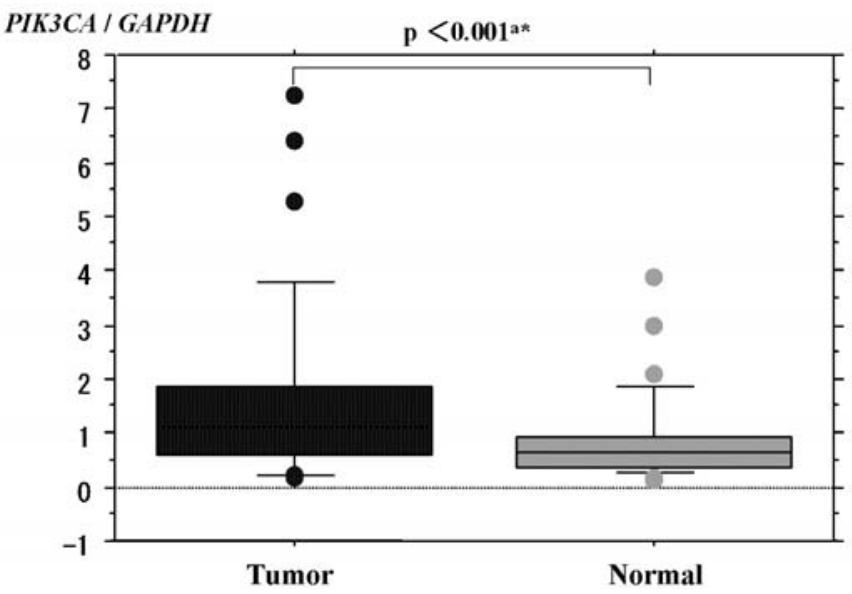

Figure 3. Quantitative real-time RT-PCR analysis of PIK3CA in 37 ESCC samples and non-tumorous esophageal epithelia. Twenty-nine of the 37 patients (78.4\%) have higher mRNA values in ESCC specimens, compared with the corresponding non-tumorous esophageal epithelia. The mean mRNA expression of PIK3CA in ESCCs was 2.61-fold higher compared with non-tumorous esophageal epithelia. ${ }^{\mathrm{a} M a n n-W h i t n e y ~ U}$ test.

with grade 0 or 1 were negative. Immunostaining was localized mainly in the cytoplasm of the tumor cells but not in their nucleus. Furthermore, comparing negative staining with positive staining for PIK3CA, the presence of node metastasis was significantly correlated with positive staining for PIK3CA (Table IV). All other clinicopathological characteristics were statistically irrelevant to positive staining for PIK3CA.

Expression of PIK3CA in ESCC cell lines. We next used immunofluorescence techniques in established human ESCC cell lines (TE-series) to confirm whether a similar pattern of PIK3CA localization existed in culture cells. In a similar manner to that observed in tumor tissues, $P I K 3 C A$ expression was observed in the cytoplasm (Fig. 5).

\section{Discussion}

Much of the complex fundamental biology of ESCC remains poorly understood, despite intensive study. One of the major aims of this study was to search for a molecular marker, which is associated with ESCC. Then, this study focuses on the analysis of molecular alterations associated with PIK3CA. In this study, we identified somatic mutations of PIK3CA in 4 out of 52 ESCC samples $(7.7 \%)$, a rate which is similar to that reported for gastric cancers $(4.3 \%)$ (25) and brain tumors (5\%) (26) but less than that observed in colorectal (18.8-31.6\%) (27) and breast (8.3-40\%) tumors (28-30). Our data were partially in line with previously reported observations (22). Our analysis was limited to exons 1, 9 and 20, therefore the frequency of genomic alteration in ESCC might have been underestimated. Obviously, there are a number of factors including great variability in the types of cancers, geographical variation/influence, sample tissue preservation and methods used for DNA isolation. This is why PIK3CA mutations are so common among colorectal and breast cancer whereas those in ESCC are uncommon. With regard to mutational spots, PIK3CA amino acid substitutions at codons 542, 545 and 1047 are the most common hot spot mutations in the various cancers. In our investigation, clean sequence traces were obtained and 4 out of 52 ESCC samples (7.7\%) were shown to harbor the A1634C change. The sensitivity of our sequencing method was proved by the faithful detection of the known differences between PIK3CA and its pseudogene (30). Along with our results, it has been reported that nearly all the mutations (21 of 24) among ovarian cancers consisted of an A1634C change in exon 9 (31). However, over 1,000 independent samples from large tumor sets identified G1624A and G1633A mutations instead of the A1634C change $(27,29,32,33)$. These studies included 229
A

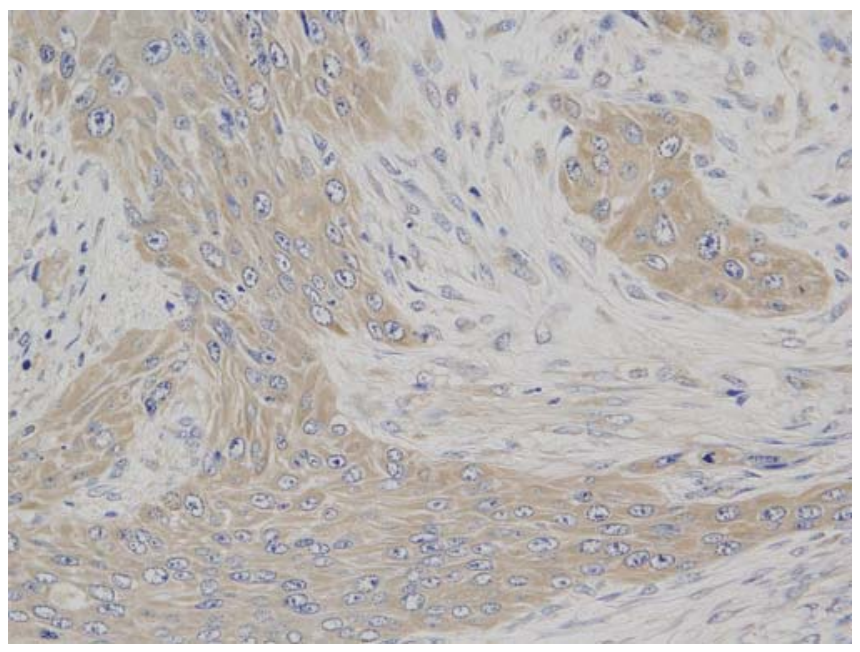

B

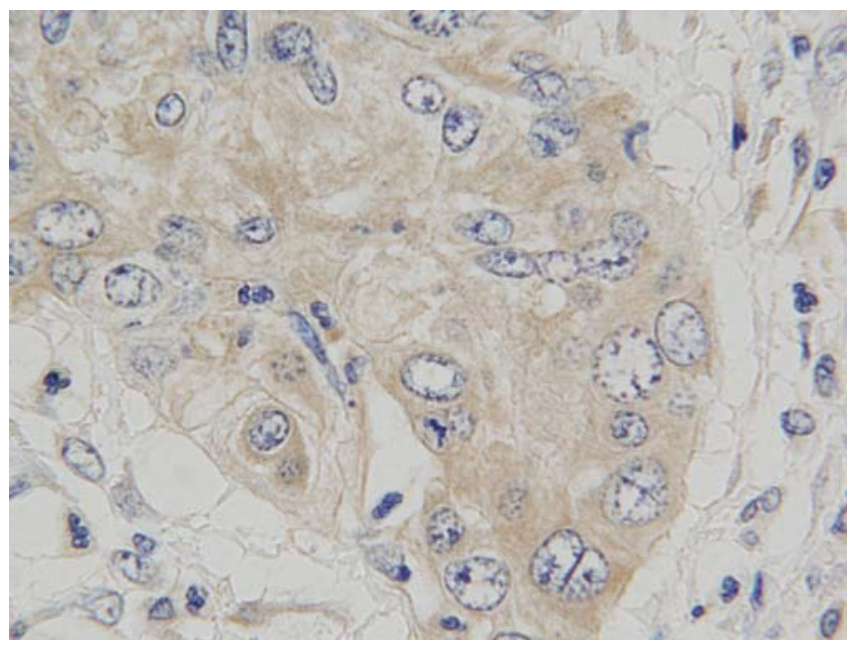

Figure 4. To examine the expression and localization of PIK3CA protein in ESCCs, we performed immunohistochemistry. Immunostaining was localized mainly in the cytoplasm of the tumor cells but not in their nucleus. The staining was scored on a scale from grade 0 to $3+$ as follows: 0 , no staining; $1+,<50 \%$ with weak or strong intensity; $2+, \geq 50 \%$ with weak intensity; $3+, \geq 50 \%$ with strong intensity. Positive immunoreactions for PIK $3 \mathrm{CA}$ were detectable in 33 of $66(50.0 \%)$ ESCC specimens with >grade 2 (original magnification: A, x400; B, x 1000), whereas the remaining 33 samples with grade 0 or 1 were defined as negative. 
Table IV. PIK3CA expression and clinicopathological factors in ESCC.

\begin{tabular}{|c|c|c|c|c|}
\hline \multirow[t]{2}{*}{ Variables } & \multicolumn{4}{|c|}{ PIK3CA expression } \\
\hline & $\mathrm{n}$ & Negative staining & Positive staining & $\mathrm{P}$-value \\
\hline \multicolumn{5}{|l|}{ Age } \\
\hline$<66$ years & 32 & 19 & 13 & $0.140^{\mathrm{a}}$ \\
\hline$\geq 66$ years & 34 & 14 & 20 & \\
\hline \multicolumn{5}{|l|}{ Gender } \\
\hline Males & 53 & 26 & 27 & $0.757^{\mathrm{a}}$ \\
\hline Females & 13 & 7 & 6 & \\
\hline \multicolumn{5}{|c|}{$\mathrm{T}$ classification } \\
\hline Tis and $\mathrm{T} 1$ & 30 & 18 & 12 & $0.138^{\mathrm{a}}$ \\
\hline $\mathrm{T} 2$ and $\mathrm{T} 3$ & 36 & 15 & 21 & \\
\hline \multicolumn{5}{|c|}{ Lymph node metastasis } \\
\hline Negative & 34 & 21 & 13 & $0.048^{\mathrm{a}, \mathrm{c}}$ \\
\hline Positive & 32 & 12 & 20 & \\
\hline \multicolumn{5}{|c|}{ Distant metastasis } \\
\hline Negative & 55 & 29 & 26 & $0.511^{\mathrm{b}}$ \\
\hline Positive & 11 & 4 & 7 & \\
\hline \multicolumn{5}{|c|}{ Vascular invasion } \\
\hline Negative & 34 & 18 & 16 & $0.622^{\mathrm{a}}$ \\
\hline Positive & 32 & 15 & 17 & \\
\hline \multicolumn{5}{|c|}{ Lymphatic invasion } \\
\hline Negative & 23 & 12 & 11 & $0.796^{\mathrm{a}}$ \\
\hline Positive & 43 & 21 & 22 & \\
\hline \multicolumn{5}{|c|}{ Intraepithelial spread } \\
\hline Negative & 43 & 19 & 24 & $0.103^{\mathrm{a}}$ \\
\hline Positive & 23 & 15 & 8 & \\
\hline \multicolumn{5}{|c|}{ Intramural metastasis } \\
\hline Negative & 60 & 31 & 29 & $0.672^{\mathrm{b}}$ \\
\hline Positive & 6 & 2 & 4 & \\
\hline \multicolumn{5}{|c|}{ Differentiation } \\
\hline Well & 30 & 17 & 13 & \\
\hline Mod & 16 & 8 & 8 & $0.513^{\mathrm{a}}$ \\
\hline Por & 20 & 8 & 12 & \\
\hline \multicolumn{5}{|l|}{ Stage } \\
\hline 0 & 4 & 3 & 1 & \\
\hline I & 19 & 9 & 10 & \\
\hline II & 20 & 10 & 10 & $0.454^{\mathrm{a}}$ \\
\hline III & 13 & 4 & 9 & \\
\hline IV & 10 & 3 & 7 & \\
\hline \multicolumn{5}{|l|}{ Recurrence } \\
\hline Negative & 50 & 28 & 22 & $0.085^{\mathrm{a}}$ \\
\hline Positive & 16 & 5 & 11 & \\
\hline
\end{tabular}

Well, well differentiated; Mod, moderately differentiated and Por, poorly differentiated. ${ }^{\mathrm{a}} \mathrm{Chi}$-square test. ${ }^{\mathrm{b}}$ Fisher's exact probability test. ${ }^{\mathrm{c}}$ The P-value is significant.

lung cancers, 185 gastric cancers, 125 breast cancers, 109 separate ovarian cancers and 234 colon cancers. Therefore, in most PIK3CA mutation studies, the mutations identified in exon 9 have almost exclusively been G1624A and G1633A changes, and to date, few studies including our own have reported the A1634C mutation (31,32,34).

Genomic aberrations of PIK3CA encompass copy number gains and amplifications or oncogenic missense mutations 


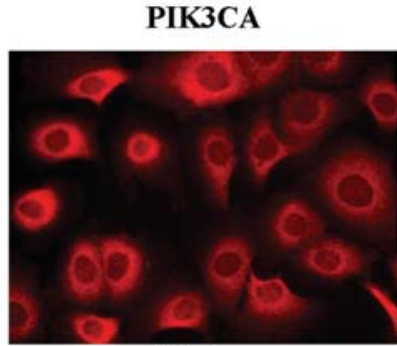

TE-1

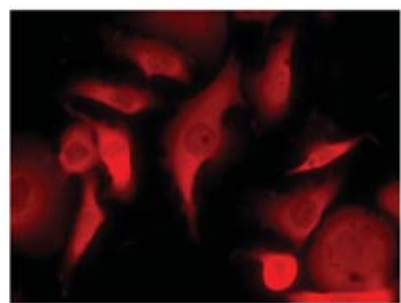

TE-8

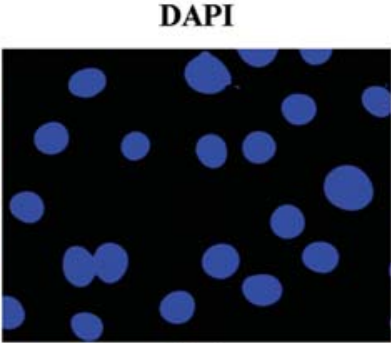

TE-1

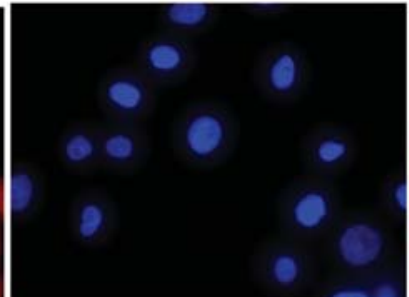

TE-8

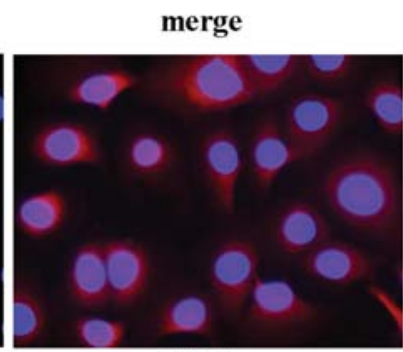

TE-1

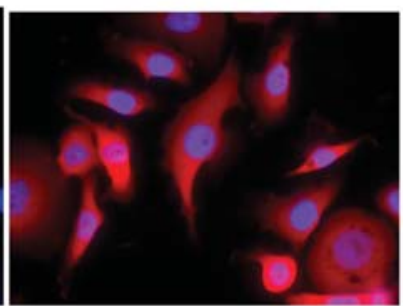

TE-8

Figure 5. Immunofluorescence was assessed in established human ESCC cell lines (TE-series) to confirm whether a similar pattern of $P I K 3 C A$ localization existed in culture cells. In a manner similar to that observed in cancer tissues, PIK3CA expression was observed in the cytoplasm.

(35-38). The copy number gains at 3q26 in ESCC, encompassing $P I K 3 C A$, have been reported previously (2). Moreover, amplification of PIK3CA in ESCC cell lines has been demonstrated by fluorescence in situ hybridization (FISH) (39). Our results showed that amplification of PIK3CA is not a common event in ESCC samples (26.7\%). However, the copy number amplification of PIK3CA detected in our data is partially connected to the polyploidy of chromosome 3 , or its short arm 3q, which are frequent events in ESCC (2). It is conceivable that overexpression of $P I K 3 C A$ in tumors is not only mediated by upstream signaling pathways but is also regulated by changes in the PIK3CA gene itself. The evidence from other previous studies suggests that the genomic gain of $P I K 3 C A$ and increased expression of $P I K 3 C A$ are associated with progression of dysplasia into invasive squamous cell carcinoma in head and neck squamous cell carcinoma (HNSCC) (40). In addition, amplification of the PIK3CA locus was shown to be a strong predictor for an early tumorassociated death in ovarian cancer (41). However, in our study, we found no statistical significance for the correlation between PIK3CA amplification and its protein expression. These data are in line with previously reported observation that overexpression of PIK3CA did not correlate with its copy number in ESCC cell lines (39). These discrepancies between overexpression and amplification show that $P I K 3 C A$ amplification is no longer specifically selected in ESCC which harbor genetic alterations, thus epigenetic events and/or other transcriptional regulation factors might influence PIK3CA expression. One possibility has been given by the inactivation of the tumor suppressor, p53, leading to enhanced PIK3CA transcription, as observed in HNSCC cell lines (42).

In PIK3CA mRNA, it has been reported that PIK3CA mRNA expression correlated with the pN status in ESCC (43). However, in this study, expression levels of mRNA were investigated only with qualitative analysis using formalin-fixed samples (43). Hence, further quantitative analyses would be considered to allow more precise outcomes. Our data using frozen samples showed that PIK3CA mRNA overexpression was highly prevalent in ESCC by quantitative RT-PCR $(\mathrm{P}<0.001)$. The vast majority of ESCC samples $(78.4 \%)$ displayed an enhanced mRNA expression but no statistically significant correlations were found between PIK3CA mRNA overexpression and any clinicopathological characteristics (data not shown).

In PIK3CA protein expression, the high rate of immunoreactive PIK3CA was supposed to be identified according to the results of PIK3CA mRNA overexpression. In our study, consistent with PIK3CA mRNA overexpression in ESCC, in just half of ESCCs $(50.0 \%)$ of immunoreactive PIK3CA was observed. The rate of immunoreactive PIK3CA in ESCC corresponded to that in lung squamous cell carcinoma (44). In a similar manner to that in tumor tissues, PIK3CA expression was observed in the cytoplasm of ESCC cell lines by immunofluorescence analysis.

Immunohistochemically in comparison with the negative staining group, the presence of node metastasis was significantly higher in the group staining positive for PIK3CA. It is certain that the protein expression is likely to be more reliable than the mRNA status because the $P I K 3 C A$ protein, not mRNA, exactly affects the transcription more directly and accurately. In our study, we failed to detect the correlation between the PIK3CA mRNA status and pN status. On the other hand, a recent study highlighted a significant correlation between the PIK3CA mRNA and $\mathrm{pN}$ status (43). The lack of association between PIK3CA mRNA and protein could be due to transcriptional or posttranscriptional mechanisms which finely control the expression level of its protein. It is possible that PIK3CA mRNA is only transiently overexpressed in tumor cells and, as a consequence, it may be difficult to detect nearly 
equivalent level of its protein. It has been reported that activation of the PI3-kinase pathway may enhance the invasion of lymph nodes by cancer cells (45) and that there is a statistically significant correlation between the presence of PIK3CA mutations and the presence of nodal metastasis in breast carcinoma (45). These findings indicate that $P I K 3 C A$ is directly involved in tumor development and that the PIK3CA gene itself may act as an oncogene (9). According to the evidence described above, PIK3CA is a putative oncogene in ESCC. Taking into consideration the fact that the patients with ESCC have a poor prognosis, it is critical to identify a population of high-risk individuals who develop ESCC rapidly. Then, the high prevalence of PIK3CA expression as a biomarker may imply the presence of lymph node metastasis in ESCC.

In conclusion, to the best of our knowledge this is the first study providing comprehensive analysis of PIK3CA in ESCC. Our results indicate that PIK3CA may play a crucial role in the development of ESCC and serve as an indicator for lymph node metastasis.

\section{Acknowledgements}

We thank Takuji Kosuge and Naoko Watanabe for their expert technical assistance. We also thank Dr T. Nishihira and Tohoku University for providing the TE-series cell lines.

\section{References}

1. Gebhart E and Liehr T: Patterns of genomic imbalances in human solid tumors (Review). Int J Oncol 16: 383-399, 2000.

2. Shinomiya T, Mori T, Ariyama Y, et al: Comparative genomic hybridization of squamous cell carcinoma of the esophagus: the possible involvement of the DPI gene in the 13q34 amplicon. Genes Chromosomes Cancer 24: 337-344, 1999.

3. Yen CC, Chen YJ, Chen JT, et al: Comparative genomic hybridization of esophageal squamous cell carcinoma: correlations between chromosomal aberrations and disease progression/ prognosis. Cancer 92: 2769-2777, 2001.

4. Tada K, Oka M, Tangoku A, Hayashi H, Oga A and Sasaki K: Gains of 8q23-qter and 20q and loss of 11q22-qter in esophageal squamous cell carcinoma associated with lymph node metastasis. Cancer 88: 268-273, 2000.

5. Pack SD, Karkera JD, Zhuang Z, et al: Molecular cytogenetic fingerprinting of esophageal squamous cell carcinoma by comparative genomic hybridization reveals a consistent pattern of chromosomal alterations. Genes Chromosomes Cancer 25: 160-168, 1999.

6. Noguchi T, Kimura Y, Takeno S, et al: Chromosomal imbalance in esophageal squamous cell carcinoma: $3 q$ gain correlates with tumor progression but not prognostic significance. Oncol Rep 10: 1393-1400, 2003.

7. Volinia S, Hiles I, Ormondroyd E, et al: Molecular cloning, cDNA sequence, and chromosomal localization of the human phosphatidylinositol 3-kinase p110 alpha (PIK3CA) gene. Genomics 24: 472-477, 1994.

8. Racz A, Brass N, Heckel D, Pahl S, Remberger K and Meese E: Expression analysis of genes at 3q26-q27 involved in frequent amplification in squamous cell lung carcinoma. Eur J Cancer 35 641-646, 1999

9. Shayesteh L, Lu Y, Kuo WL, et al: PIK3CA is implicated as an oncogene in ovarian cancer. Nat Genet 21: 99-102, 1999.

10. Ma YY, Wei SJ, Lin YC, et al: PIK3CA as an oncogene in cervical cancer. Oncogene 19: 2739-2744, 2000.

11. Redon R, Muller D, Caulee K, Wanherdrick K, Abecassis J and du Manoir S A: Simple specific pattern of chromosomal aberrations at early stages of head and neck squamous cell carcinomas: PIK3CA but not p63 gene as a likely target of 3q26-qter gains. Cancer Res 61: 4122-4129, 2001.
12. Vivanco I and Sawyers CL: The phosphatidylinositol 3-Kinase AKT pathway in human cancer. Nat Rev Cancer 2: 489-501, 2002.

13. Klippel A, Escobedo MA, Wachowicz MS, et al: Activation of phosphatidylinositol 3-kinase is sufficient for cell cycle entry and promotes cellular changes characteristic of oncogenic transformation. Mol Cell Biol 18: 5699-5711, 1998.

14. Khwaja A, Rodriguez-Viciana P, Wennstrom S, Warne PH and Downward J: Matrix adhesion and Ras transformation both activate a phosphoinositide 3-OH kinase and protein kinase B/Akt cellular survival pathway. EMBO J 16: 2783-2793, 1997.

15. Kennedy SG, Wagner AJ, Conzen SD, et al: The PI 3-kinase/ Akt signaling pathway delivers an anti-apoptotic signal. Genes Dev 11: 701-713, 1997 .

16. Frevert EU and Kahn BB: Differential effects of constitutively active phosphatidylinositol 3-kinase on glucose transport, glycogen synthase activity, and DNA synthesis in 3T3-L1 adipocytes. Mol Cell Biol 17: 190-198, 1997.

17. Cantley LC: The phosphoinositide 3-kinase pathway. Science 296: 1655-1657, 2002.

18. Guo XN, Rajput A, Rose R, et al: Mutant PIK3CA-bearing colon cancer cells display increased metastasis in an orthotopic model. Cancer Res 67: 5851-5858, 2007.

19. Ikenoue T, Kanai F, Hikiba Y, et al: Functional analysis of PIK3CA gene mutations in human colorectal cancer. Cancer Res 65: 4562-4567, 2005.

20. Kang S, Bader AG and Vogt PK: Phosphatidylinositol 3-kinase mutations identified in human cancer are oncogenic. Proc Natl Acad Sci USA 102: 802-807, 2005.

21. Bader AG, Kang S and Vogt PK: Cancer-specific mutations in PIK3CA are oncogenic in vivo. Proc Natl Acad Sci USA 103: 1475-1479, 2006

22. Phillips WA, Russell SE, Ciavarella ML, et al: Mutation analysis of PIK3CA and PIK3CB in esophageal cancer and Barrett's esophagus. Int J Cancer 118: 2644-2646, 2006.

23. Fenic I, Steger K, Gruber C, Arens C and Woenckhaus J: Analysis of PIK3CA and Akt/protein kinase B in head and neck squamous cell carcinoma. Oncol Rep 18: 253-259, 2007.

24. Kim TJ, Lee JW, Song SY, et al: Increased expression of pAKT is associated with radiation resistance in cervical cancer. $\mathrm{Br} \mathrm{J}$ Cancer 94: 1678-1682, 2006.

25. Li VS, Wong CW, Chan TL, et al: Mutations of PIK3CA in gastric adenocarcinoma. BMC Cancer 5: 29, 2005

26. Broderick DK, Di C, Parrett TJ, et al: Mutations of PIK3CA in anaplastic oligodendrogliomas, high-grade astrocytomas, and medulloblastomas. Cancer Res 64: 5048-5050, 2004.

27. Samuels Y, Wang Z, Bardelli A, et al: High frequency of mutations of the PIK3CA gene in human cancers. Science 304: 554,2004

28. Samuels Y and Velculescu VE: Oncogenic mutations of PIK3CA in human cancers. Cell Cycle 3: 1221-1224, 2004.

29. Bachman KE, Argani P, Samuels Y, et al: The PIK3CA gene is mutated with high frequency in human breast cancers. Cancer Biol Ther 3: 772-775, 2004.

30. Campbell IG, Russell SE, Choong DY, et al: Mutation of the PIK3CA gene in ovarian and breast cancer. Cancer Res 64: 7678-7681, 2004.

31. Levine DA, Bogomolniy F, Yee CJ, et al: Frequent mutation of the PIK3CA gene in ovarian and breast cancers. Clin Cancer Res 11: 2875-2878, 2005

32. Lee JW, Soung YH, Kim SY, et al: PIK3CA gene is frequently mutated in breast carcinomas and hepatocellular carcinomas. Oncogene 24: 1477-1480, 2005.

33. Wang Y, Helland A, Holm R, Kristensen GB and Borresen-Dale AL: PIK3CA mutations in advanced ovarian carcinomas. Hum Mutat 25: 322,2005

34. Hartmann C, Bartels G, Gehlhaar C, Holtkamp N and von Deimling A: PIK3CA mutations in glioblastoma multiforme. Acta Neuropathol (Berl) 109: 639-642, 2005

35. Qiu W, Schonleben F, Li X, et al: PIK3CA mutations in head and neck squamous cell carcinoma. Clin Cancer Res 12: 1441-1446, 2006.

36. Kozaki K, Imoto I, Pimkhaokham A, et al: PIK3CA mutation is an oncogenic aberration at advanced stages of oral squamous cell carcinoma. Cancer Sci 97: 1351-1358, 2006.

37. Yen CC, Chen YJ, Pan CC, et al: Copy number changes of target genes in chromosome 3q25.3-qter of esophageal squamous cell carcinoma: TP63 is amplified in early carcinogenesis but down-regulated as disease progressed. World J Gastroenterol 11: $1267-1272,2005$ 
38. Pedrero JM, Carracedo DG, Pinto CM, et al: Frequent genetic and biochemical alterations of the PI 3-K/AKT/PTEN pathway in head and neck squamous cell carcinoma. Int J Cancer 114: 242-248, 2005

39. Imoto I, Pimkhaokham A, Fukuda Y, et al: SNO is a probable target for gene amplification at $3 \mathrm{q} 26$ in squamous-cell carcinomas of the esophagus. Biochem Biophys Res Commun 286: 559-565, 2001.

40. Woenckhaus J, Steger K, Werner E, et al: Genomic gain of PIK3CA and increased expression of p110alpha are associated with progression of dysplasia into invasive squamous cell carcinoma. J Pathol 198: 335-342, 2002.

41. Woenckhaus J, Steger K, Sturm K, Munstedt K, Franke FE and Fenic I: Prognostic value of PIK3CA and phosphorylated AKT expression in ovarian cancer. Virchows Arch 450: 387-395, 2007
42. Singh B, Reddy PG, Goberdhan A, et al: p53 regulates cell survival by inhibiting PIK3CA in squamous cell carcinomas. Genes Dev 16: 984-993, 2002.

43. Wada S, Noguchi T, Takeno S and Kawahara K: PIK3CA and TFRC located in $3 \mathrm{q}$ are new prognostic factors in esophageal squamous cell carcinoma. Ann Surg Oncol 13: 961-966, 2006

44. Lin X, Bohle AS, Dohrmann P, et al: Overexpression of phosphatidylinositol 3-kinase in human lung cancer. Langenbecks Arch Surg 386: 293-301, 2001.

45. Saal LH, Holm K, Maurer M, et al: PIK3CA mutations correlate with hormone receptors, node metastasis, and ERBB2, and are mutually exclusive with PTEN loss in human breast carcinoma. Cancer Res 65: 2554-2559, 2005. 